\title{
Magnetic Resonance Imaging (MRI) Based on Machine Learning Algorithms for the Diagnosis in Efficacy of Dexmedetomidine along with Modified Electroconvulsive Therapy Nursing on First Episode Schizophrenia
}

\author{
Qingyan Zhao $\mathbb{D}^{1}$ and Shuai Yuan $\mathbb{D}^{2}$ \\ ${ }^{1}$ School of Nursing, Shaoyang University, Shaoyang 422000, Hunan, China \\ ${ }^{2}$ Department of Anesthesiology, The First Affiliated Hospital of Shaoyang Uniuersity, Shaoyang 422000, Hunan, China
}

Correspondence should be addressed to Shuai Yuan; 9915001@caa.edu.cn

Received 21 September 2021; Revised 4 November 2021; Accepted 6 November 2021; Published 29 November 2021

Academic Editor: M. Pallikonda Rajasekaran

Copyright ( 2021 Qingyan Zhao and Shuai Yuan. This is an open access article distributed under the Creative Commons Attribution License, which permits unrestricted use, distribution, and reproduction in any medium, provided the original work is properly cited.

\begin{abstract}
This study was aimed to explore the efficacy of dexmedetomidine combined with modified electroconvulsive therapy (MECT) and continuous nursing on first episode schizophrenia (FES), as well as the continuous monitoring effect of magnetic resonance imaging (MRI) on the FES patients under machine learning algorithms. In this study, 48 schizophrenia patients who received the combined treatment of MECT and dexmedetomidine were selected, and they were divided into the routine group (RG) and observation group (OG), with routine nursing and continuous nursing, respectively. Besides, another 30 healthy people were included as the control group (Ctrl group). Based on MRI characteristics, the machine learning algorithm designed for FES patients was used to analyze the changes in the amplitude low-frequency fluctuations (ALFF) of the static state brain MRI; the positive and negative score scale (PANSS) was used to evaluate the mental symptoms of patients. The Schizophrenia Quality of Life Scale (SQLS) score, treatment compliance rate, follow-up rate, and recurrence rate of patients were also compared. The results showed that the image quality of the weakened and enhanced areas of brain MRI was significantly improved under the machine learning algorithm. After treatment, the total score of PANSS and the scores of all dimensions were greatly reduced $(P<0.05)$. For the FES patients, the ALFF value of the frontal right lower lobe before treatment was higher than that of the Ctrl group $(P<0.05)$, while the ALFF value of the right insula and right superior frontal gyrus after treatment was lower than that of the Ctrl group $(P<0.01)$, and the ALFF value of the left inferior frontal gyrus opercular part was lower than that of the Ctrl group $(P<-0.05)$. The SQLS score, treatment compliance rate, follow-up rate, and recurrence rate of OG after nursing intervention were all much higher than those of the Ctrl group $(P<-0.05)$. In summary, MRI based on machine learning algorithms could be applied to monitor the effect of dexmedetomidine combined with MECT, and continuous nursing made an effective improvement in SQLS score of FES patients, though which the risk of recurrence was reduced.
\end{abstract}

\section{Introduction}

Schizophrenia is mainly a mental illness in which patients have a variety of dysfunctions in their thinking, behavior, mood, and spirit. According to relevant statistics, its lifetime prevalence is about $4.0-8.4 \%$, and it occurs mostly in young adults, which has a great impact on daily life and work [1].
Patients usually have hallucinations and delusions and hurt others or themselves. About $10 \%$ of patients will commit suicide and self-injury [2]. Modified electroconvulsive therapy (MECT) is a physical therapy method with rapid onset, which has good clinical effects in the treatment of schizophrenia, unipolar, bipolar depression, and other mental diseases $[3,4]$. MECT mainly stimulates brain cells 
through impulse currents and then induces extensive EEG production, thereby regulating neurotransmitters in the brain to alleviate patients' mental symptoms.

With traditional electroconvulsive therapy, patients may have symptoms such as trance, nausea, headache, and jaw pain after treatment. These symptoms may last for several hours and are gradually improved within a few weeks without any treatment. There are also other side effects, such as muscle pain and short-term or long-term impaired memory. Less infrequent side effects may include increased blood pressure, changes in heart rhythm, and convulsions for longer time than expected [5]. The symptoms of cerebral cortex discharge caused by MECT are very similar to those of epileptic seizures, so MECT may bring side effects such as muscle tonic spasm and fractures in patients [6].

Propofol is usually used as an intravenous anesthetic to relieve the symptoms of convulsions and panic psychology [7]. However, recent studies have shown that when propofol is combined with MECT treatment, the probability of seizure threshold increases, the twitch time is correspondingly reduced, and the seizure intensity is greatly decreased, which has a greater impact on the final treatment effect [8]. Studies have indicated that dexmedetomidine hydrochloride can reduce the effect of stress response, has a high a 2 adrenergic receptor selectivity, exerts a good sedative, hypnotic, and analgesic effect, and requires less intravenous anesthetics [9]. At present, there are more reports on the treatment of hemodynamics by dexmedetomidine and MECT, but there are few studies on patients with schizophrenia, and the efficacy of dexmedetomidine and MECT in the treatment of schizophrenia still remains to be evaluated.

There are many kinds of brain function imaging techniques for mental illness such as electroencephalogram, positron emission tomography, and functional MRI (fMRI) $[10,11]$. fMRI has the advantages of high resolution, noninvasiveness, and simple and easy operation, which can clearly display the functional activity status of specific areas of the brain and is the most widely used clinically [12]. Static state fMRI is currently the preferred method for monitoring brain function changes in MECT treatment of schizophrenia. Traditional group analysis methods are mainly aimed at a certain functional network in the human brain, but recent studies have shown that some brain regions can have influence in multiple functional networks at the same time.

In recent years, the rapid development of machine learning and pattern recognition provides new ideas for the analysis and activation detection of fMRI signals. Convolutional sparse coding algorithm has been widely applied in many fields at present, and it has shown good processing effects in image deblurring, super-resolution reconstruction, and image denoising of medical images [13]. Sparse representation can fully show the differences between groups, and the introduction of machine learning algorithms into sparse coding methods is beneficial to analyze the differences between patients and healthy volunteers. Some studies have pointed out that the further optimization of convolutional sparse coding algorithm can be realized, by solving the convolution least squares approximation with the conjugate gradient descent method or relaxing the equality constraints with the continuation method. It is also reported that a fast convolutional sparse coding algorithm based on the block cycle feature of the cyclic block matrix in the Fourier domain is proposed, which can also achieve image optimization. Similarly, the convolutional sparse coding algorithm can be improved with the alternating direction method of multipliers (ADMM) [14].

The sparse coding algorithm under machine learning was used to process fMRI images of FES patients in this study, for the evaluation of dexmedetomidine combined with MECT on FES and the effect of continuous nursing. The thinking was expanded for the optimization of the clinical treatment of FES, and some reference values of the sparse coding algorithm in fMRI image optimization were offered.

\section{Materials and Methods}

2.1. Research Subjects and Grouping. 48 FES patients who stayed in our hospital from July 2018 to July 2019 were selected as the research subjects, and 30 healthy volunteers during the same period were rolled into the Ctrl group. Inclusion criteria I: aged 18-45 years old, right-handed; II: disease duration not more than three years, and systemic medication duration not more than one year; III: meeting the Diagnostic and Statistical Manual of Mental Disorders Fifth Edition ((DSM) -5) diagnostic criteria; IV: PANSS score greater than (inclusive) 69 points; V: meeting the indications of MECT and not accepting MECT treatment in the past six months. Exclusion criteria I: those who were contraindicated with magnetic resonance; II: those who had a history of severe head trauma; III: those who had a family history of epilepsy; IV: those who had suicidal behavior and cannot cooperate with the examination; V: those who were pregnant or breast feeding. This experiment had obtained permission from the ethics committee of the hospital, and all the patients included were informed and agreed to it.

\subsection{Combined Therapy of MECT and Dexmedetomidine.} Six hours before the treatment, the patients were water-free and fasted, and urine and bowels were emptied. Then, the active denture was taken out, and the chest radiograph, electrocardiogram, and blood routine were checked. Before anesthesia, $0.5 \mu \mathrm{g} / \mathrm{kg}$ dexmedetomidine solution (containing $20 \mathrm{ml} 0.9 \%$ normal saline) was pumped for about 10 minutes. After that, $0.5 \mathrm{mg}$ atropine was injected, and propofol was injected until the eyelash reflex disappeared. Subsequently, $0.8 \mathrm{mg} / \mathrm{kg}$ succinylcholine chloride was injected, and assisted breathing was performed. When the symptoms of bi-digital fibrillation disappeared completely, the patient's muscles gradually relaxed, and MECT treatment was performed. Seizures were induced and assisted breathing was conducted during MECT treatment. After the patient's spontaneous breathing recovered, they needed to wait in the resuscitation room for vital signs to stabilize and then returned to the ward. The MECT instrument was adopted for treatment, and the stimulation power was $2 / 3$ of the patient's age, and the changes in electrocardiogram, blood pressure, and blood oxygen saturation were 
monitored. The PANSS was applied to assess mental symptoms, and the clinical efficacy was evaluated according to the reduction rate: reduction rate $=\mid$ total score difference before and after treatment//total score before treatment. $75 \%-100 \%, 50 \%-74 \%, 25 \%-49 \%$, and $0-25 \%$ corresponded to cured, markedly effective, effective, and ineffective, respectively.

2.3. Magnetic Resonance Detection in the Resting State. In this study, the MRI imaging system was introduced to scan the patient's head, and the patient's head was fixed. The collection of the blood oxygen level was dependent on BOLD signal, and the patient was in resting state during the scan. First, anatomical positioning image scan and high-resolution three-dimensional structure image scan were performed, respectively, followed by fMRI scan in the resting state. The T1 FLAIR and GRE-EPI sequences were used for BOLD scan. The fMRI scan flip angle in the resting state was $30^{\circ}$, the field of view was $220 \times 220 \mathrm{~mm}^{2}$, the ratio of pulse repetition time to echo time was $2000 / 30 \mathrm{~ms}$, the matrix was $64 \times 64$, the layer thickness was $4 \mathrm{~mm}$, and the layer spacing was $0.8 \mathrm{~mm}$. The functional magnetic resonance was applied to scan for 10 minutes to obtain 24 consecutive time point images, and DPARSFA2.3 software was adopted to preprocess fMRI image data. The ALFF value was obtained using REST software.

2.4. MRI Images Based on Machine Learning Algorithms. First, the MRI data were preprocessed, and the basic process is shown in Figure 1. Among them, the calculation method of head movement is as follows:

$$
\frac{S}{R}=\frac{1}{m-1} \sum_{i=2}^{m} \sqrt{\left|x_{i}-x_{i-1}\right|^{2}-\left|y_{i}-y_{i-1}\right|^{2}+\left|z_{i}-z_{i-1}\right|^{2}},
$$

where $x i, y i$, and $z i$ represented the ratio of displacement and rotation in the direction of $x, y$, and $z$ at time point $i$.

In this study, machine learning algorithms were introduced into the sparse representation method, and a group sparse coding algorithm based on machine learning was proposed to process MRI, and the algorithm flow is shown in Figure 2. For the main process of the algorithm, various image signals in the MRI image were firstly extracted and transferred into the two-dimensional signal matrix $D x$. Then, the image information was further processed through the signal matrix $D$, the dictionary matrix, and the coefficient matrix. Finally, the processed image information was transferred to the group sparse map.

2.4.1. fMRI Signal Extraction. After the MRI image was preprocessed, signal extraction was required. The nonbrain area was removed from the image after signal extraction, which was convenient for subsequent image analysis. First, the image signal was mapped to the MNI space; then, the standard brain template was adopted to match the time point brain image, and the fMRI signal was extracted. If $x$ was the fMRI signal of the sample to be tested, then $D x$ is the vector formed by the signal intensity of the voxel at the corresponding time point (Figure 2).

2.4.2. Building of a Group Analysis Matrix. The fMRI signals of the sample $x$ were arranged to obtain a two-dimensional signal matrix $D_{x} \in R^{t \times n_{x}}, t$ was the time point, and $n x$ was the voxel number. Then, dictionary learning was conducted to get the signal matrix $D \in R^{t \times n}$, as shown in Figure 2:

$$
\begin{aligned}
D & =\left[D_{A}, D_{B}\right], \\
D_{A} & =\left[D_{a 1}, D_{a 2}, D_{a 3}, \ldots, D_{a k}\right], \\
D_{B} & =\left[D_{b 1}, D_{b 2}, D_{b 3}, \ldots, D_{b k}\right],
\end{aligned}
$$

where the subscripts $A$ and $B$ represent the Ctrl group and FES patients, respectively, and $a k$ and $b k$ are the number of the two groups.

\subsubsection{Dictionary Learning and Sparse Representation.} Matrix $D$ was decomposed into coefficient matrix $\alpha$ and dictionary matrix T. $\alpha=\left[\alpha_{A}, \alpha_{B}\right] \in R^{m \times n} ; n$ was the total number of voxels, and $m$ was the number of dictionaries. If $D=\left[D_{1}, D_{2}, D_{3}, \ldots, D_{k}\right] \in R^{t \times n}$, the dictionary $T$ can be expressed as $T \in R^{t \times m}(m>t, m \ll n)$ (Figure 2). According to the prior loss function $\mathrm{fn}(\mathrm{T})$, the average expression error was expressed, and the $\varphi$ norm was used for constraint:

$$
\begin{aligned}
D & =T E+\varepsilon, \\
f_{n}(T) & \triangleq \\
n & \sum_{i=1}^{n} \varphi\left(s_{i}, T\right), \\
\varphi\left(s_{i}, T\right) & \triangleq \min _{\alpha \in R^{m}} \frac{1}{2}\left\|s_{i}-T \alpha_{i}\right\|_{2}^{2}+\lambda\left\|\alpha_{i}\right\|_{1} .
\end{aligned}
$$

Then, normalization was carried out, and $g_{1}, g_{2}, g_{3}, \ldots g_{m}$ column was constrained according to equation (4), and the signal residuals obeyed the standard distribution $\varepsilon_{\mathrm{i}} \sim N\left(0, \sigma^{2}\right)$ :

$$
F \triangleq\left\{T \in R^{t \times m} \text { s.t. } \forall j=1, \ldots, m g_{j}^{H} g_{j} \leq 1\right\} .
$$

The above process can be summarized by the following equation:

$$
\min _{T \in F, \alpha \in R^{m \times n}} \frac{1}{2}\|D-T E\|_{G}^{2}+\lambda\|\alpha\|_{1,1} .
$$

2.4.4. Group Coefficient Mapping. The obtained coefficient matrix $\alpha$ needed to obtain the voxel position information in the corresponding human brain according to map, and finally, the difference between healthy volunteers and schizophrenia patients was discussed. The resistivity mapping and analysis methods used are shown in Figure 3. 


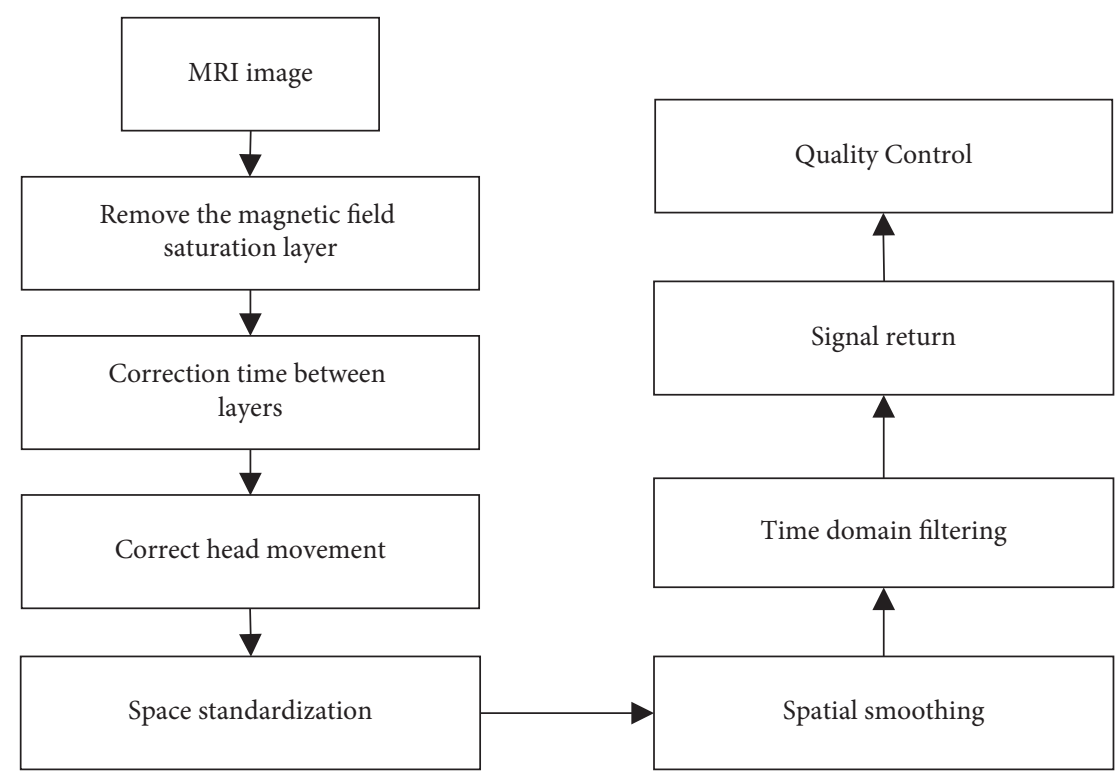

FIgURE 1: MRI image data preprocessing.

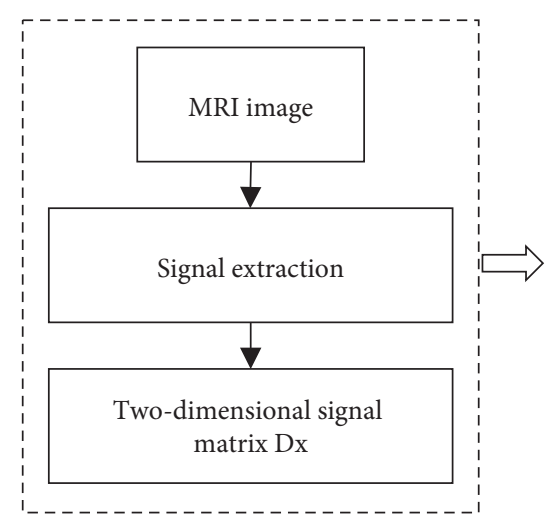

(1)

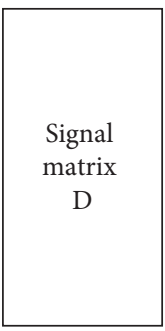

(2)

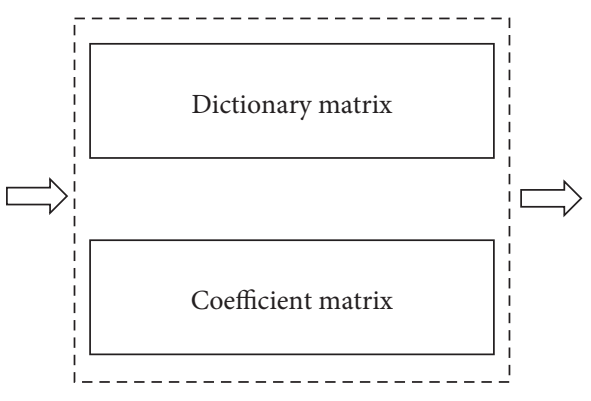

(3)

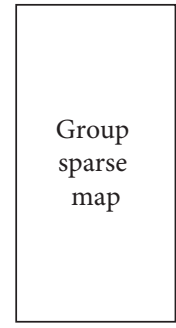

(4)

FIGURE 2: Group sparse coding algorithm of the FMRI image.

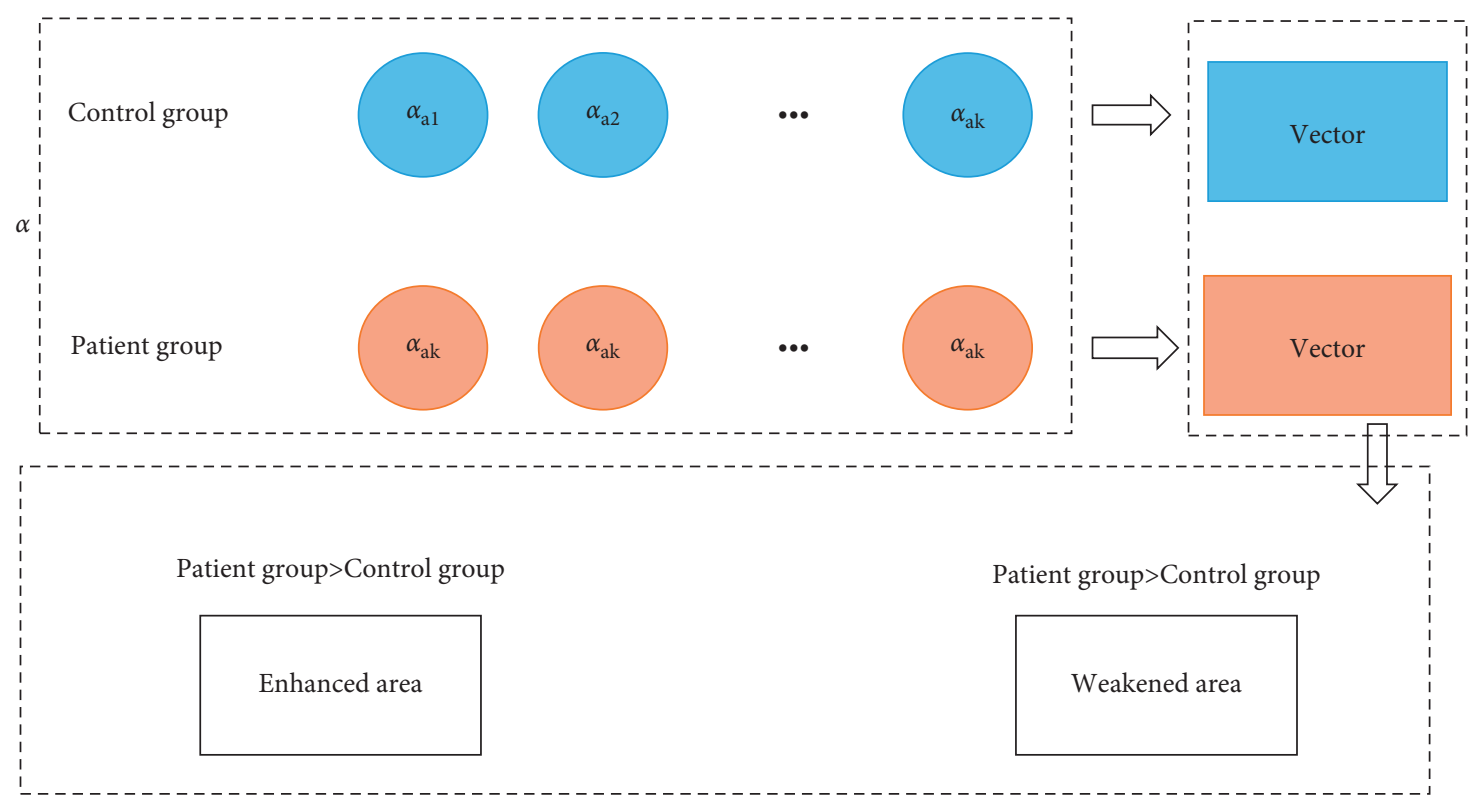

FIGURE 3: Group coefficient mapping method. 
2.5. Nursing Methods. FES patients were rolled into RG and OG in random. SQLS was adopted to assess the SQLS score of the subjects after 6 months.

Continuing nursing: a special nursing team was set up, telephone follow-up was performed once a week, and family visit was performed once a month; it was necessary to pay attention to changes in the patient's mental state during nursing and give necessary psychological counseling treatment according to the actual situation; the patient should be supervised to take medication on time, and detailed medication guidance should be given; patients should be guided to develop a good lifestyle and encouraged to exercise outdoors to improve their immunity; timely understanding the patient's condition was needed and patients should be advised to have regular follow-up visits.

2.6. Statistical Analysis. The experimental data of this study were processed by SPSS20.0. The measurement data were represented by $(\bar{x} \pm s)$, which stood for mean \pm standard deviation. The comparison of the means between groups was performed by $t$-test, the enumeration data were expressed as a percentage (\%), and the $\chi^{2}$ test was used for the verification. When $P<0.05$, the difference was considered to be statistically significant.

\section{Results}

3.1. General Data of the Patients. As shown in Table 1, the general information of the FES patients and healthy volunteers were collected.

3.2. Detection Results of Machine Learning Algorithm. MRI technology based on machine learning algorithms was adopted to detect the images of FES patients and the Ctrl group on the COBRE dataset. Double-sample T-test was used to obtain the enhanced and weakened activity areas of FES patients in contrast with healthy people, and the results were shown in Figures 4 and 5.

3.3. Comparison of Mental Status. In contrast with before treatment, the total score of PANSS and the scores of each dimension were greatly reduced after the combined treatment, $P<0.05$ (Figure 6). The reduction rate was further calculated, which was greater than $25 \%$ after treatment, and there were 17 with markedly effective results and 31 with effective results.

3.4. Changes of ALFF Value in the Resting State. The brain regions with significant differences in ALFF before and after MECT treatment in FES patients were in contrast with healthy examiners, and the results are shown in Figure 7. After treatment, the ALFF value of the right insula of FES patients was greatly lower than the Ctrl group $(P<0.01)$. Before treatment, the ALFF values of the right inferior lobules, the left inferior frontal gyrus, and the right superior frontal gyrus of FES patients were greatly lower than the Ctrl group $(P<0.01)$.
The correlation between ALFF values of the right insula and the right parietal inferior lobule and PANSS score was not significant $(P>0.05)$. There was an obvious negative correlation between the ALFF value of the left inferior frontal gyrus and aggressive hostility $(P<0.05)$. ALFF values of the right superior frontal gyrus were negatively correlated with positive symptoms and total scores $(P<0.05)$, as shown in Table 2 .

3.5. Comparison of the Nursing Effect. The treatment compliance rate, follow-up rate, and recurrence rate of FES patients in the OG were greatly higher than the Ctrl group, $P<0.05$, as shown in Table 3 . Before nursing intervention, there was no obvious difference in the scores and total scores of the RG and the OG $(P>0.05)$; after the intervention, the scores and total scores of the OG were greatly lower than the RG $(P<0.05)$, as shown in Figure 8.

\section{Discussion}

In this study, machine learning algorithms were applied to MRI image processing, and the results suggested that it can clearly distinguish the complex brain function differences between healthy volunteers and FES patients, which was consistent with the research results of Kerepesi et al. [15], indicating the machine learning algorithm can improve the accuracy of the MRI signal detection results, which had great reference value for monitoring the efficacy of dexmedetomidine combined with MECT treatment. Then, $\mathrm{fMRI}$ images were included to analyze brain changes in FES patients, and it was found that, before treatment, the ALFF value of the right parietal lobule was higher than the Ctrl group, which was similar to the research results of Suzuki et al. [16] and Chen et al. [17], indicating that the brain area was active here, while ALFF in the other three places was elevated, but it was not statistically significant; in contrast with before treatment, brain activity in the four brain regions treated by dexmedetomidine combined with MECT was reduced. The four brain regions monitored are located around the frontal lobe-limbic system-parietal nerve anatomical loop, and studies have shown that this loop participates in the pathogenesis of schizophrenia [18], indicating that MECT may have affected the activity intensity of brain neurons in this part, thereby inhibiting psychosis.

The PANSS scale is usually used to evaluate the treatment effect of patients with schizophrenia. It was found in the study that the total score of PANSS and the scores of various dimensions in patients with FES were greatly reduced after treatment, $P<0.05$, which was consistent with the results of Hansbauer et al. [19], indicating that dexmedetomidine combined with MECT therapy can effectively improve the brain function of patients with FES and have a certain antipsychotic effect. Mental diseases have a greater impact on the physical, psychological, and social functions of patients, which seriously harms the SQLS score of patients [20]. In the study, continuation nursing was applied, and it was found that, after nursing intervention, the FES patients in OG had a higher follow-up rate and treatment compliance 
TABle 1: Comparison of baseline data.

\begin{tabular}{|c|c|c|c|}
\hline & Health examination personnel $(n=30)$ & FES patients $(n=48)$ & $P$ \\
\hline Gender: male/female & $20 / 10$ & $32 / 16$ & 0.564 \\
\hline Age (year) & $32.34 \pm 12.87$ & $34.12 \pm 11.63$ & 0.876 \\
\hline Weight $(\mathrm{kg})$ & $61.76 \pm 10.79$ & $64.36 \pm 11.38$ & 0.675 \\
\hline Years of education (years) & $12.89 \pm 2.34$ & $11.98 \pm 3.12$ & 0.153 \\
\hline Course of disease (years) & - & $8.73 \pm 6.59$ & - \\
\hline
\end{tabular}

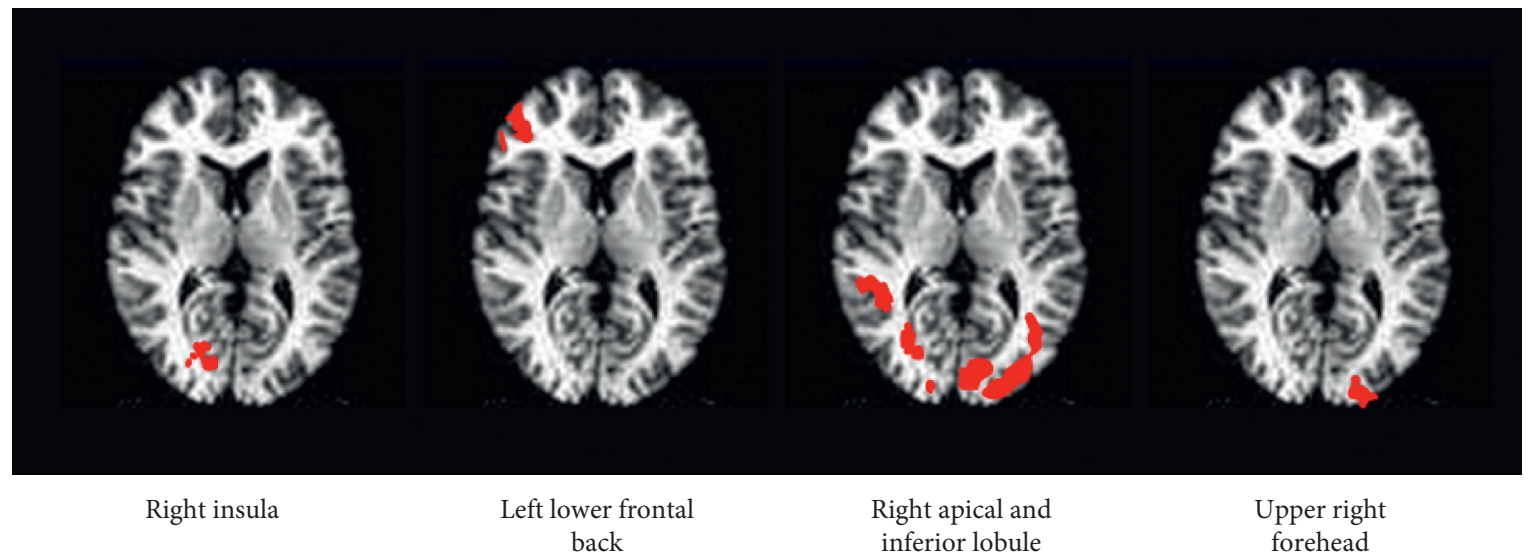

FIGURE 4: MRI brain enhancement area (red area).

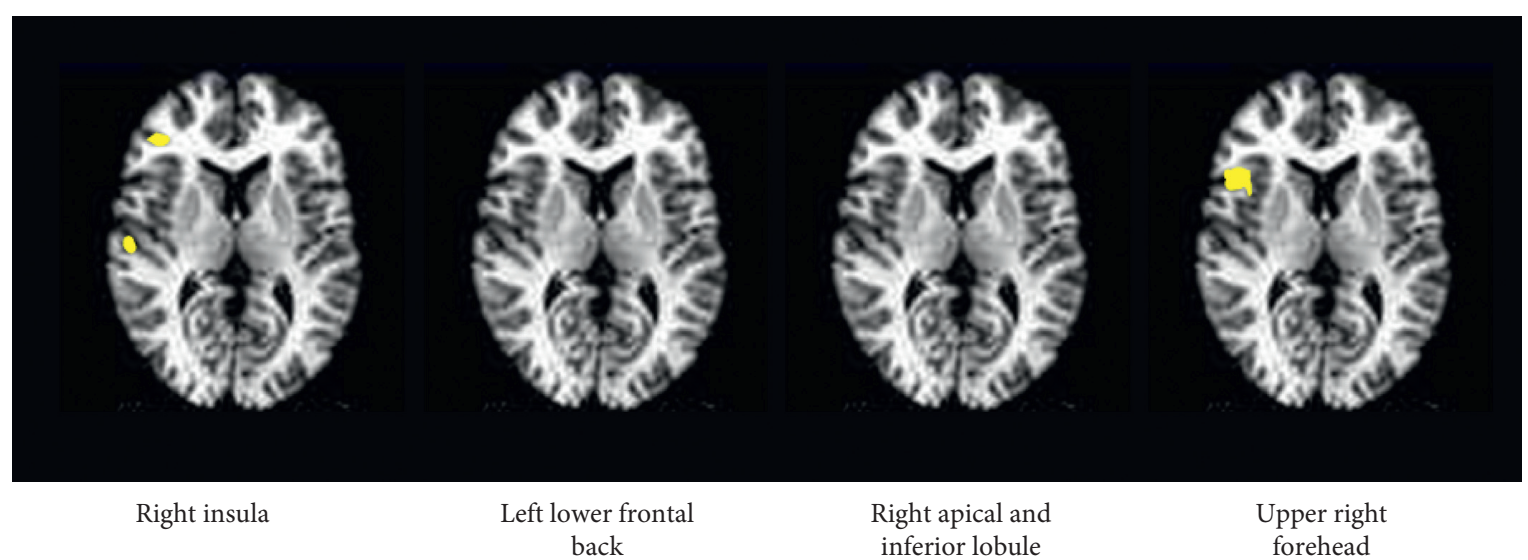

FIGURE 5: MRI brain weakened area (yellow area).

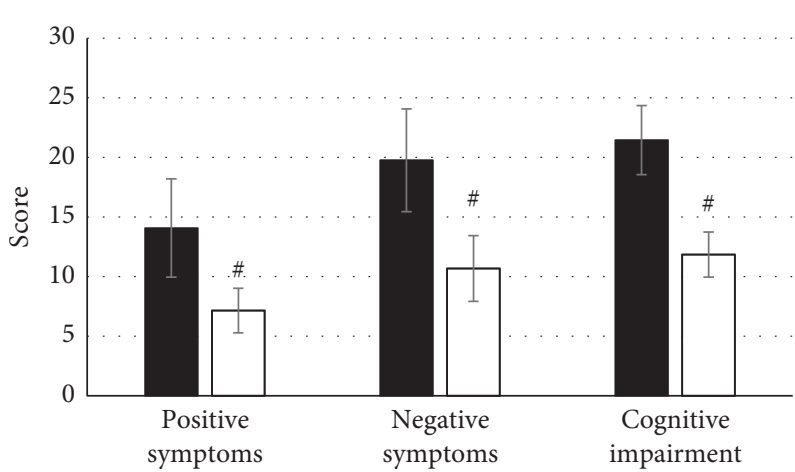

- Before treatment

$\square$ After treatment

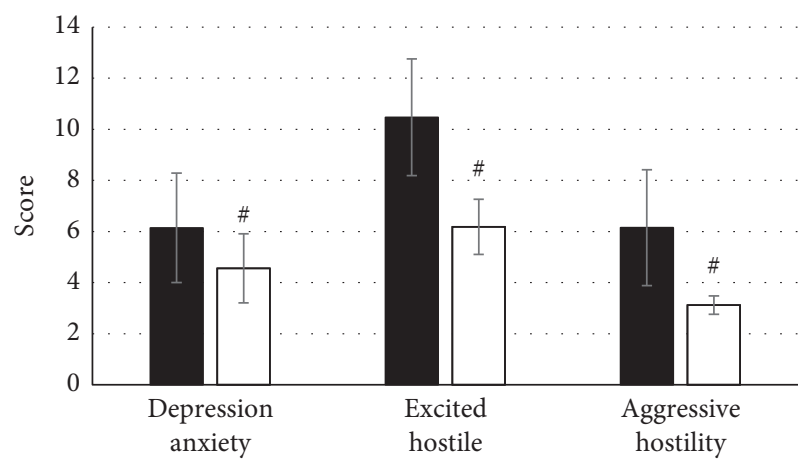

- Before treatment

․ After treatment

(a) 


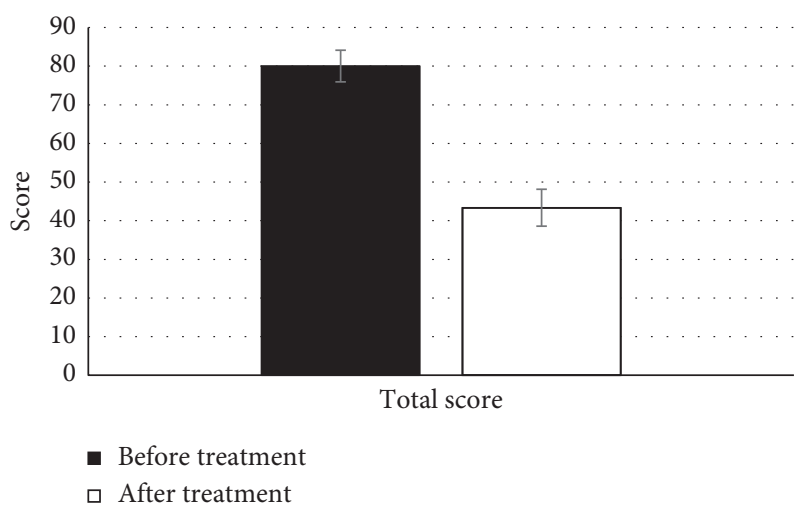

(c)

FIgURE 6: Comparison of PANSS scores and total scores (A is positive symptom, negative symptom, and cognitive impairment; B is depressed anxiety, excited hostility, and aggressive hostility; ${ }^{\#}$ indicated that, in contrast with the RG, $P<0.01$ ).

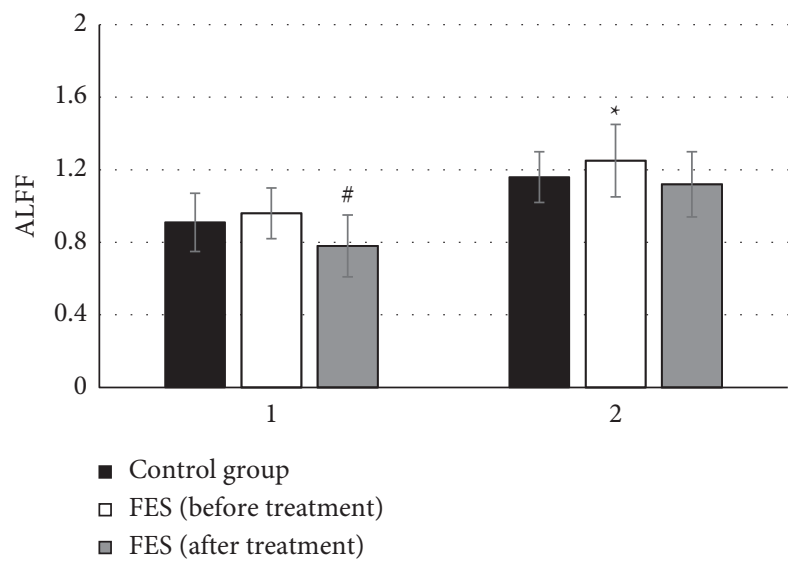

(a)

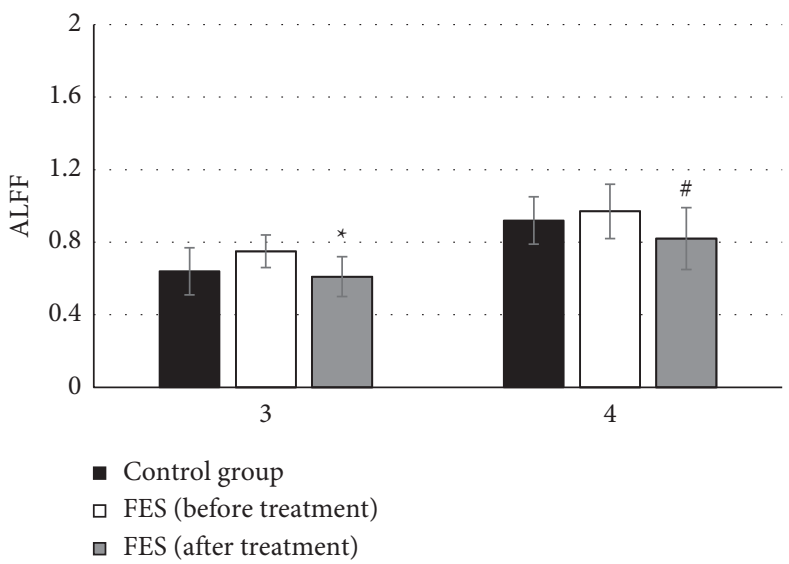

(b)

Figure 7: Changes of the ALFF value in the resting state (A was the right insula; $B$ was the right parietal inferior lobule; $C$ was left inferior frontal gyrus opercular part; $\mathrm{D}$ was the right superior frontal gyrus; * indicated that, in contrast with the RG, $P<0.05$; ${ }^{*}$ indicated that, in contrast with the RG, $P<0.01)$.

TABLE 2: Correlation between ALFF value and PANSS score.

\begin{tabular}{|c|c|c|c|}
\hline ALFF value & PNASS scale & $t$ & $P$ \\
\hline \multirow{7}{*}{ Left inferior frontal gyrus opercular part } & Positive symptoms & 0.345 & 0.675 \\
\hline & Negative symptoms & 0.123 & 0.187 \\
\hline & Cognitive impairment & 0.989 & 0.652 \\
\hline & Depressed anxiety & 0.340 & 0.126 \\
\hline & Excited hostility & -0.108 & 0.348 \\
\hline & Aggressive hostility & -0.369 & 0.039 \\
\hline & Total score & -0.342 & 0.128 \\
\hline \multirow{7}{*}{ Right superior frontal gyrus } & Positive symptoms & -0.267 & 0.028 \\
\hline & Negative symptoms & 0.324 & 0.057 \\
\hline & Cognitive impairment & 1.262 & 1.762 \\
\hline & Depressed anxiety & 3.762 & 3.762 \\
\hline & Excited hostility & 1.982 & 0.076 \\
\hline & Aggressive hostility & -0.267 & 0.675 \\
\hline & Total score & -0.324 & 0.047 \\
\hline
\end{tabular}


TABLE 3: Comparison of intervention effects.

\begin{tabular}{|c|c|c|c|c|}
\hline & Ctrl group & OG & $t$ & $P$ \\
\hline Treatment compliance rate $(\%)$ & 66.23 & 72.67 & 2.762 & 0.031 \\
\hline Follow-up rate (\%) & 52.78 & 71.89 & 1.892 & 0.008 \\
\hline Recurrence rate $(\%)$ & 12.65 & 0.98 & 1.076 & 0.029 \\
\hline
\end{tabular}

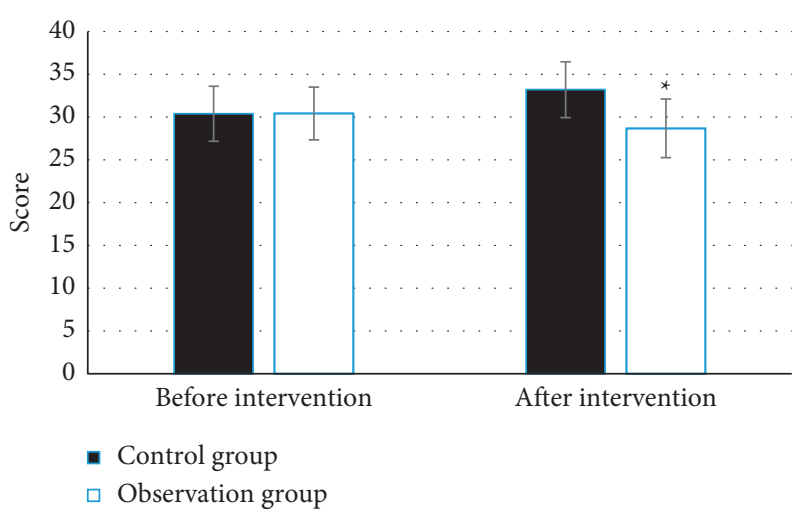

(a)

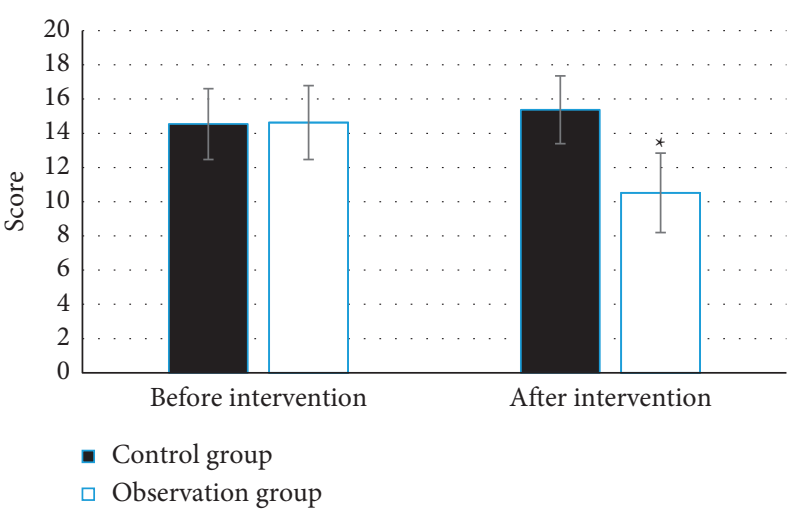

(b)

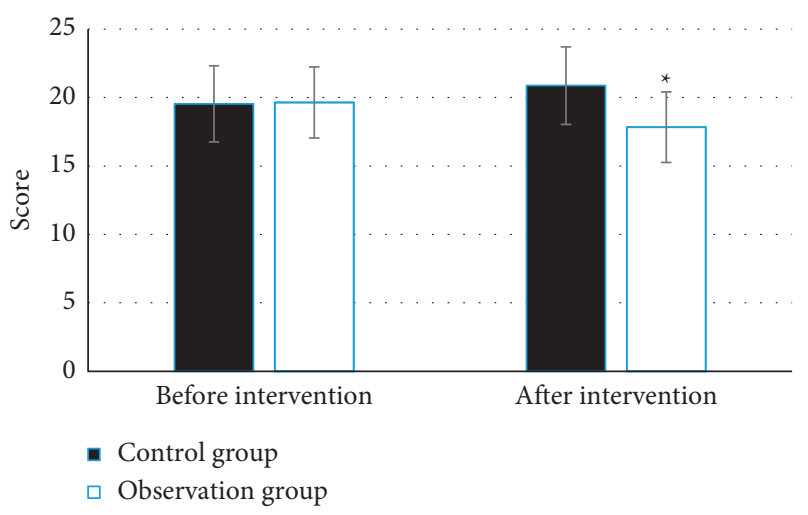

(c)

FIgURE 8: Comparison of SQLS score (A is psychology; B is energy motivation; C is symptoms and adverse reactions; ${ }^{*}$ means that, in contrast with the RG, $P<0.05)$.

rate and a lower recurrence rate. The dimensional scores of SQLS improved, indicating that the continuous nursing was beneficial to improve the SQLS score of patients and lower the risk of recurrence.

\section{Conclusion}

MRI based on machine learning algorithms can be used to monitor the efficacy of dexmedetomidine combined with MECT in the treatment of FES, and continuous nursing can improve the SQLS of FES patients. However, the number of samples in this study is limited, and it has not been compared with drug treatment or other treatment methods. In the future, it is necessary to increase the number of samples and compare it with other methods to further explore the effect of the combined treatment of dexmedetomidine and MECT on the brain function of FES patients. In short, the results can provide a theoretical basis to clarify the pathogenesis of schizophrenia and optimize treatment interventions of FES.

\section{Data Availability}

The data used to support the findings of this study are available from the corresponding author upon request.

\section{Conflicts of Interest}

The authors declare no conflicts of interest.

\section{References}

[1] A. F. Pardiñas, P. Holmans, A. J. Pocklington et al., "Common schizophrenia alleles are enriched in mutation-intolerant genes and in regions under strong background selection," Nature Genetics, vol. 50, no. 3, pp. 381-389, 2018.

[2] C. Hjorthøj, A. E. Stürup, J. J. McGrath, and M. Nordentoft, "Years of potential life lost and life expectancy in schizophrenia: a systematic review and meta-analysis," Lancet Psychiatry, vol. 4, no. 4, pp. 295-301, 2017.

[3] Q. Chen, J. Dong, J. Luo et al., "Effects of low-dose ketamine on the antidepressant efficacy and suicidal ideations in 
patients undergoing electroconvulsive therapy," The Journal of ECT, vol. 36, no. 1, pp. 25-30, 2020.

[4] K. R. Abraham and P. Kulhara, "The Efficacy of Electroconvulsive Therapy in the Treatment of Schizophrenia," British Journal of Psychiatry, vol. 151, no. 2, pp. 152-155, 2018.

[5] C. Andrade, S. S. Arumugham, and J. Thirthalli, "Adverse effects of electroconvulsive therapy," Psychiatric Clinics of North America, vol. 39, no. 3, pp. 513-530, 2016.

[6] S. C. Kaliora, I. M. Zervas, and G. N. Papadimitriou, "[Electroconvulsive therapy: 80 years of use in psychiatry]," Psychiatriki, vol. 29, no. 4, pp. 291-302, 2018.

[7] M. Nuzzi, D. Delmonte, B. Barbini et al., "Thiopental is better than propofol for electroconvulsive therapy," Acta biomedica: Atenei Parmensis, vol. 88, no. 4, pp. 450-456, 2018.

[8] S. Shah, I. Chowdhury, A. Bhargava, and B. Sabbharwal, "Comparison of hemodynamic effects of intravenous etomidate versus propofol during induction and intubation using entropy guided hypnosis levels," Journal of Anaesthesiology Clinical Pharmacology, vol. 31, no. 2, pp. 180-185, 2015.

[9] Y. Tonooka and K. Sunada, "Dexmedetomidine enhances the pulpal anesthetic effect of lidocaine: a pilot study," Anesthesia Progress, vol. 65, no. 1, pp. 38-43, 2018.

[10] S. Shin, S. Kim, S. Seo et al., "The relationship between dopamine receptor blockade and cognitive performance in schizophrenia: a [11C]-raclopride PET study with aripiprazole," Translational Psychiatry, vol. 8, no. 1, p. 87, 2018.

[11] Q. Zhu, J. Huang, and X. Xu, "Non-negative discriminative brain functional connectivity for identifying schizophrenia on resting-state fMRI," BioMedical Engineering Online, vol. 17, no. 1, p. 32, 2018.

[12] A. Juneja, B. Rana, and R. K. Agrawal, "fMRI based computer aided diagnosis of schizophrenia using fuzzy kernel feature extraction and hybrid feature selection," Multimedia Tools and Applications, vol. 77, no. 3, pp. 3963-3989, 2018.

[13] R. M. Chesnut, N. Temkin, S. Dikmen et al., "A-method-ofmanaging severe-traumatic.brain-injury-in the-absence-of intracranial-pressure-monitoring. the-imaging and.clinical-examination protocol," I-Neurotrauma, vol. 35 , no. 1 , pp. 54-63, 2018.

[14] C.-N. Li, Y.-H. Shao, W. Yin, and M.-Z. Liu, "Robust and sparse linear discriminant analysis via an alternating direction method of multipliers," IEEE Transactions on Neural Networks and Learning Systems, vol. 31, no. 3, pp. 915-926, 2020.

[15] C. Kerepesi, B. Szalkai, B. Varga, and V. Grolmusz, "Comparative connectomics: mapping the inter-individual variability of connections within the regions of the human brain," Neuroscience Letters, vol. 662, pp. 17-21, 2018.

[16] H. Suzuki, R. Enatsu, A. Kanno et al., "The involvement of sensory-motor networks in reflex seizure," NMC Case Report Journal, vol. 4, no. 4, pp. 127-130, 2017.

[17] Y. Chen, R. Wang, B. Hou et al., "Regional brain activity during rest and gastric water load in subtypes of functional dyspepsia: a preliminary brain functional magnetic resonance imaging study," Journal of Neurogastroenterology and Motility, vol. 24, no. 2, pp. 268-279, 2018.

[18] M. Popescu, J. D. Hughes, E.-A. Popescu et al., "Activation of dominant hemisphere association cortex during naming as a function of cognitive performance in mild traumatic brain injury: insights into mechanisms of lexical access," NeuroImage: Clinical, vol. 15, pp. 741-752, 2017.

[19] M. Hansbauer, T. Wobrock, B. Kunze et al., "Efficacy of highfrequency repetitive transcranial magnetic stimulation on PANSS factors in schizophrenia with predominant negative symptoms - results from an exploratory re-analysis," Psychiatry Research, vol. 263, pp. 22-29, 2018.

[20] A. Rayan and K. Obiedate, "The correlates of quality of life among Jordanian patients with schizophrenia," Journal of the American Psychiatric Nurses Association, vol. 23, no. 6, pp. 404-413, 2017. 